\title{
Estudio comparativo radiológico de los parámetros cardiacos entre imágenes alineadas y con desviación en la vista dorsoventral del tórax
}

\section{Comparative radiological study of cardiac parameters between lined up and rotated dorsoventral view of the thorax}

\author{
Bianca M. Baldi Aste ${ }^{1}$, Jesús Chilón ${ }^{1}$, Isaac Chipayo ${ }^{1}$, Diego Díaz C. ${ }^{1}$
}

\section{RESUMIEN}

El objetivo de este estudio fue comparar los parámetros cardiacos de radiografías de tórax de vista dorsoventral alineada con los de vista dorsoventral girada de un mismo paciente para determinar el grado de giro aceptable para dicha vista. Se utilizaron 53 placas dorsoventrales giradas de 32 pacientes caninos y se compararon los parámetros de dichas placas con sus respectivas muestras control o placas dorsoventrales alineadas. Para ello, se usaron las pruebas de analogía del reloj y relación cardio-torácica y complementadas con su respectiva vista latero-lateral derecha, permitiendo la obtención del ángulo de giro. Los resultados de las muestras giradas fueron asignadas a un grupo de $0.1^{\circ}$ a $6^{\circ}$ de giro y a otro de $6^{\circ}$ a $12^{\circ}$ de giro. Cada cámara cardiaca fue evaluada con su respectiva placa control mediante la prueba de McNemar. El grupo de $0.1^{\circ}$ a $6^{\circ}$ de giro presenta un valor de probabilidad de 0.1025 al evaluar la aurícula derecha, 0.6547 para la aurícula izquierda, 0.1573 para el ventrículo derecho y 0.3173 para el ventrículo izquierdo, siendo válido para diagnóstico; a diferencia del grupo de $6^{\circ}$ a $12^{\circ}$ de giro que obtuvo 0.0196 para la aurícula derecha, 0.0578 para la aurícula izquierda, 0.0114 para el ventrículo derecho y 0.0126 para el ventrículo izquierdo, no siendo aceptables para diagnóstico.

Palabras clave: caninos radiografía, dorsoventral, corazón

\footnotetext{
${ }^{1}$ Clínica de Animales Menores, Facultad de Medicina Veterinaria, Universidad Nacional Mayor de San Marcos, Lima, Perú

${ }^{2}$ E-mail: ichipayo@hotmail.com
}

Recibido: 13 de marzo de 2018

Aceptado para publicación: 20 de diciembre de 2019

Publicado: 31 de marzo de 2020 
The aim of this study was to compare the cardiac parameters of chest X-rays with aligned dorsoventral view with those of the rotated dorsoventral view of the same patient to determine the acceptable degree of rotation for these views. Fifty-three rotated dorsoventral plates from 32 canine patients were used and the parameters compared of those plates with their respective control samples or aligned dorsoventral plates. To do this, the clockface analogy test and the cardio-thoracic ratio were used and complemented with their respective right side-lateral view, allowing obtaining the angle of rotation. The results of the rotated samples were assigned to a group of $0.1^{\circ}$ to $6^{\circ}$ of rotation and to another group of $6^{\circ}$ to $12^{\circ}$ of rotation. Each cardiac chamber was evaluated with its respective control plate by the $\mathrm{McNemar}$ test. The group from $0.1^{\circ}$ to $6^{\circ}$ rotation presented a probability value of 0.1025 when evaluating the right atrium, 0.6547 for the left atrium, 0.1573 for the right ventricle and 0.3173 for the left ventricle, being valid for diagnosis; unlike the group of $6^{\circ}$ to $12^{\circ}$ rotation that obtained 0.0196 for the right atrium, 0.0578 for the left atrium, 0.0114 for the right ventricle and 0.0126 for the left ventricle, not being acceptable for diagnosis.

Key words: canine, radiograph, dorsoventral, heart

\section{INTRODUCCIÓN}

La radiografía es una imagen registrada en una película fotográfica, que se obtiene al exponer un objeto a una fuente de alta energía. En el caso del diagnóstico por imágenes, los rayos $\mathrm{X}$ dan una imagen interna del objeto bajo evaluación en una escala de grises, dependiendo de su composición, mediante la transformación de energía electromagnética en energía química (Bushong, 2013).

Desde su descubrimiento de los Rayos X en 1895 por Wilhelm Conrad Röentgen, ha tenido una función importante en medicina para el diagnóstico de diversos tipos de enfermedades y patologías, entre ellas las cardiacas y respiratorias (Bushong, 2013), y su uso ha sido adoptado y adaptado en medicina veterinaria. Uno de los inconvenientes con la realización de radiografías de tórax en medicina veterinaria es la dosis de radiación a la que se expone tanto el radiólogo o tec- nólogo al tener que repetir en numerosas ocasiones una vista radiológica, con el objetivo de obtener la mejor vista (Hartung, 1992; Massey, 1971; Álvarez et al., 2007), dado que la posición y sujeción del paciente le genera estrés, comprometiendo su capacidad respiratoria (Tilley et al., 2008; Atkins et al., 2009).

En el país, la entidad designada para realizar la autorización, control y fiscalización de instalaciones que realizan uso de radiación ionizante es el Instituto Peruano de Energía Nuclear (IPEN), el cual se rige a la Norma Técnica IR.003.2013 para el registro de exposiciones médicas (IPEN, 2013).

El presente estudio tuvo como objetivo determinar los parámetros cardiacos de radiografías de tórax dorsoventrales no alineadas (las vértebras torácicas no se superponen a las esternebras perfectamente) y su comparación con los parámetros cardiacos de placas de tórax dorsoventrales alineadas. 


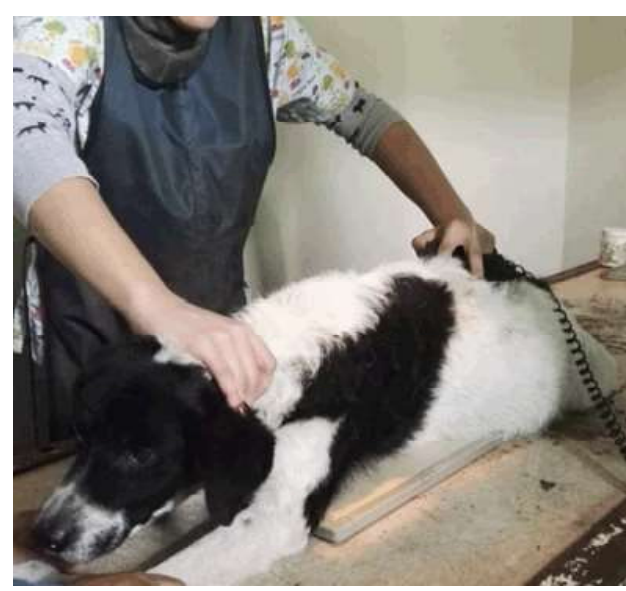

Figura 1. Posicionamiento del paciente canino sin sedación para obtención de vista radiográfica dorsoventral

\section{MATERIALes y Métodos}

\section{Lugar de Estudio}

El estudio se realizó en la Sala de Radiología de la Clínica de Animales Menores (CAM) de la Facultad de Medicina Veterinaria (FMV) de la Universidad Nacional Mayor de San Marcos (UNMSM), Lima, Perú, entre junio y diciembre de 2016.

\section{Toma de Placas de Rayos X}

Todos los pacientes que participaron en el estudio necesitaron las proyecciones radiográficas del tórax latero lateral derecha (LLD) y dorsoventral (DV). Los parámetros cardiológicos de la proyección LLD no fueron evaluados, pero permitieron hallar el ángulo de giro correspondiente a la proyección DV no alineada, además de permitir realizar una evaluación completa del tórax como parte del diagnóstico radiológico rutinario.

Para la toma de placa radiográfica en proyección DV, los animales fueron colocados sobre la mesa de rayos $\mathrm{X}$ sin ser sedados, se determinó el grosor de la cavidad torácica y se les sujetó para colocarlos en posición decúbito esternal (Figura 1). Para el presente estudio se incluyeron todas aquellas imágenes que provinieron de caninos con aumento cardiaco según la proyección de tórax $\mathrm{DV}$ alineada y que hayan requerido la repetición de dicha proyección debido a que se tuvo la necesidad de obtener una proyección DV no alineada. Además, se excluyeron las placas radiográficas que resultaron distorsionadas $\mathrm{o}$ borrosas, donde los márgenes cardiacos y los bordes óseos no estuvieron bien delimitados o definidos. Se trabajó con la máquina de rayos X Mobile 250 General Electric, películas radiográficas médicas verdes (MXG de 8x10, $9.5 \times 12$ y $11 \times 14 "$ Carestream, USA) y revelador y reforzador manual GBX (Carestream, USA). Los tiempos usados fueron los establecidos para el procesamiento manual de radiografías (Thrall, 2012). Las medidas se hicieron con una regla milimetrada de $30 \mathrm{~cm}$.

El tamaño muestral mediante la fórmula de poblaciones finitas, con un error muestral de 0.05 , nivel de confianza de $95 \%$ y valor de probabilidad a favor de $50 \%$, teniendo en cuenta si el paciente gira o no gira, se obtuvo mediante la siguiente fórmula: $\mathrm{n}=\left(\mathrm{z}^{2}\right.$. $\mathrm{p}$. $\mathrm{q}$. $\mathrm{N}) /\left(\mathrm{e}^{2}(\mathrm{~N}-1)+\mathrm{z}^{2} \cdot \mathrm{p} \cdot \mathrm{q}\right)$, donde $\mathrm{n}=$ tamaño muestral, $\mathrm{z}=$ coeficiente de nivel de confian$\mathrm{za}, \mathrm{p}=$ probabilidad a favor, $\mathrm{q}=$ probabilidad en contra, $\mathrm{N}=$ población de pacientes caninos que requieren radiografías de tórax, $\mathrm{e}=$ error máximo permisible (Aguilar, 2005). El número de pacientes requeridos fue de 53 , lo cual se obtuvo en siete meses de trabajo.

\section{Toma y Evaluación de Placas}

Las placas radiográficas de tórax fueron tomadas en la proyección DV, considerando la placa DV no alineada (posicionamiento inadecuado debido a que las vértebras y las esternebras no se encuentran alineadas, pero los márgenes anatómicos son definidos de manera que si permite hacer mediciones) como grupo muestral, y la proyección DV alineada como grupo control, de manera que ambas tomas provinieron de un mismo paciente. 
Cada paciente fue colocado sobre la mesa de rayos $\mathrm{X}$, donde se procedió con un examen clínico incluyendo la frecuencia cardiaca. Luego fue recostado sobre su lado derecho encima del chasis y se realizó la toma LLD, vista que complementa la toma DV en el estudio cardiológico, y que además sería usada para hallar el ángulo de giro. Luego se procedió a tomar la vista DV colocando al paciente en posición decúbito esternal sobre el chasis. La colimación y los $\mathrm{kVp}$ usados con cada paciente, dependieron de la distancia entre la zona dorsal y ventral del tórax que correspondería a la altura, y del del espesor de este. En varias ocasiones, se requirió tomar varias placas hasta poder obtener la muestra control.

Para la lectura de resultados o evaluación de la placa radiográfica con proyección latero lateral derecha (LLD) se usaron los métodos de evaluación VHS (Buchanan, 2000) y para las vistas DV se usaron los métodos de analogía del reloj. La relación cardiotorácica (entre 50 a $60 \%$ del tórax en un canino aparentemente normal) según Tilley et al. (2008).

Las proyecciones DV obtenidas fueron evaluadas individualmente, teniendo en cuenta los siguientes criterios:

- Distancia entre la $6^{\text {ta }}$ vértebra torácica y $6^{\text {ta }}$ esternebra, según toma LLD

- VHS (Vertebral Heart Size)

- Medidas cardiacas (ancho, largo)

- Medidas del tórax (ancho, distancia entre los márgenes de cada hemitórax y el margen cardiaco correspondiente. Todas las medidas fueron realizadas a nivel de las zonas cardiacas que presentaban aumento)

- Distancia entre la $6^{\text {ta }}$ vértebra torácica y $6^{\text {ta }}$ esternebra, según toma DV no alineada

- Ángulo de giro de placa DV no alineada

- Diagnósticos (placa DV alineada [Control] y DV no alineada)

- Ubicación del desplazamiento

- Tipo de tórax
Los datos fueron agrupados de acuerdo al rango de giro y fueron evaluados para cada cámara del corazón por separado.

\section{Evaluación de la proyección latero late- ral derecha}

Se evalúan campos pulmonares en busca de alguna alteración y se toma la medida a nivel del punto central del $6^{\text {to }}$ cuerpo vertebral hacia la $6^{\text {ta }}$ esternebra, debido a que representa el punto central del corazón, tanto en la vista LLD, como en la vista DV. De esta manera se obtiene el lado (h), valor que fue usado para determinar el grado del ángulo de giro del tórax en la proyección DV. Además, se procedió a determinar el valor del VHS (Buchanan, 2000), como valor referencial y parte de la evaluación completa de las placas de tórax.

\section{Evaluación de las proyecciones dorso- ventrales}

Se tomaron medidas del largo y ancho del corazón, y del ancho del tórax. Así mismo, las diferencias entre cada lado del corazón y cada hemitórax, a nivel de las regiones donde había aumento cardiaco en sus puntos más amplios. Luego se tomaron las medidas de las distancias entre las esternebras y las vértebras en las tomas DV mal alineadas o giradas a nivel del punto central del cuerpo vertebral de la $6^{\text {ta }}$ vértebra torácica y de la $6^{\text {ta }}$ esternebra.

Posteriormente se examinó cada placa DV, considerando el método de la analogía del reloj y la relación cardio-torácica. Se mide el largo y ancho del corazón, ancho del tórax y la distancia entre el corazón y los márgenes de cada hemitórax (Figura 2). Esto permite clasificar según el tipo de tórax en perros de tórax estrecho, normal o en barril como se observa (Figura 3) (Kittleson y Kienle, 2000). Finalmente, se procedió a anotar los diagnósticos por separado de las placas DV alineadas y las placas DV mal alineadas o giradas. 


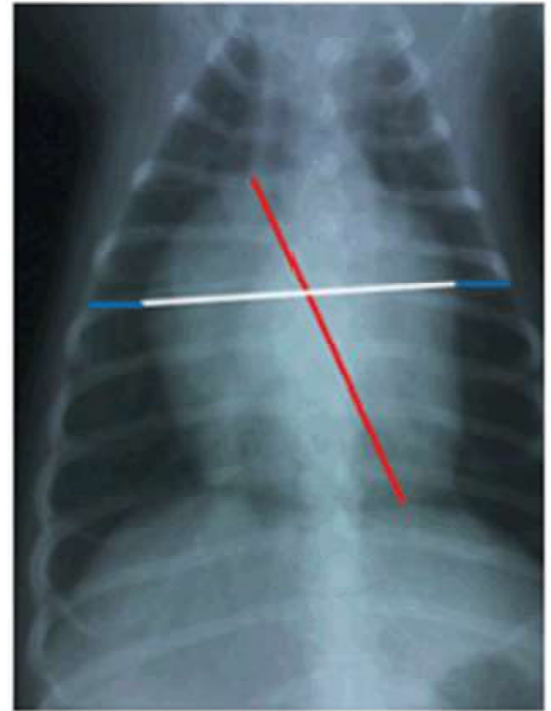

A

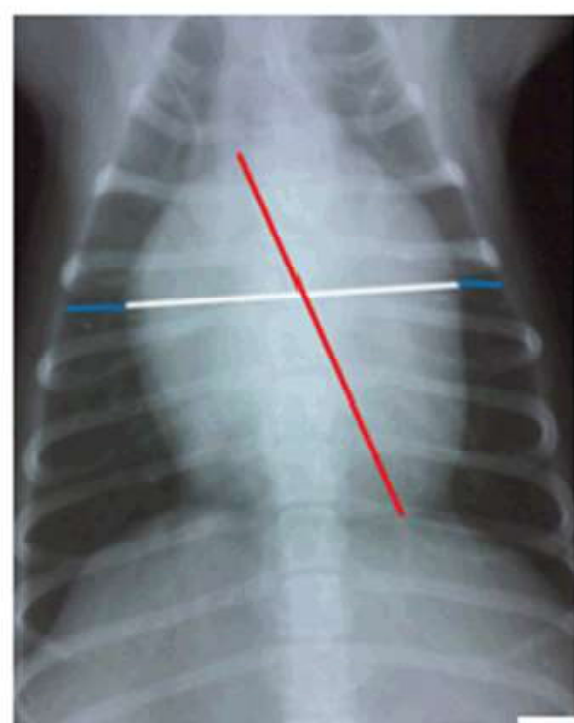

B

Figura 2. Toma de medidas de ancho y largo del corazón y distancias de los puntos más anchos del corazón hacia los márgenes de cada hemitórax en perros en la vista dorsoventral no alineada (A) y dorsoventral alineada - Control (B)

\section{Diagnóstico radiológico}

Una vez obtenidos los datos, se realizó el diagnóstico de la placa control en conjunto con la toma LLD, así como con los datos del paciente. Luego se hizo el diagnóstico de la radiografía DV girada o mal alineada en conjunto con la toma LLD (una única toma LLD es usada para hacer el diagnóstico de ambas tomas DV).

\section{Cálculo del ángulo de giro}

El método utilizado para calcular el ángulo de giro de la vista DV no alineada en el presente estudio es una variable del método usado por Hamlin (1968) para calcular el ángulo de giro. Esta variable se desarrolló en la CAM para el presente estudio, teniendo como referencia que las medidas a utilizar son las más cercanas al tamaño real del tórax estudiado, puesto que las proyecciones realizadas se obtuvieron con la zona irradiada en permanente contacto con el chasis usado.
Adicionalmente, este método toma en cuenta los tres tipos de tórax.

Se obtiene tomando como referencia las siguientes medidas:

- La distancia vértebra-esternebra tomada desde el centro de la $6^{\text {ta }}$ vértebra torácica hasta el centro de la $6^{\text {ta }}$ esternebra, obtenida en la toma LLD $(\mathrm{H})$.

- La distancia vértebra-esternebra tomada desde el centro de la $6^{\text {ta }}$ vértebra torácica hasta el centro de la $6^{\text {ta }}$ esternebra obtenida en la toma DV no alineada o girada (A).

donde $(\mathrm{H})$ sería la hipotenusa del triángulo formado por esas distancias, y (A) sería el cateto opuesto al ángulo de giro. De manera que, para hallar el cateto adyacente (B) al ángulo de giro, se recurre a la fórmula: $\mathrm{H}^{2}=$ $\mathrm{A}^{2}+\mathrm{B}^{2}$. Una vez obtenido el cateto opuesto, se obtiene el valor de la tangente del triángulo formado por dichos valores mediante la función tangente de Excel (TAN), función 


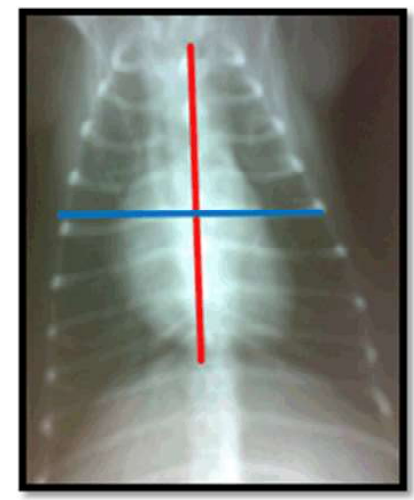

A

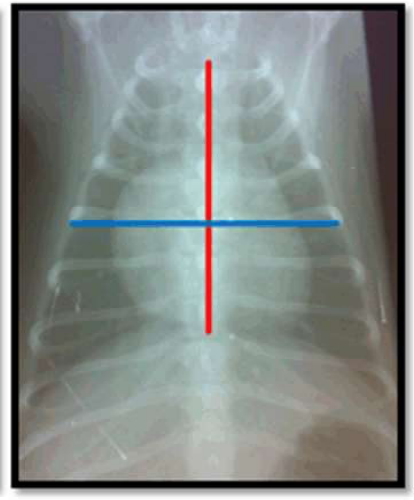

B

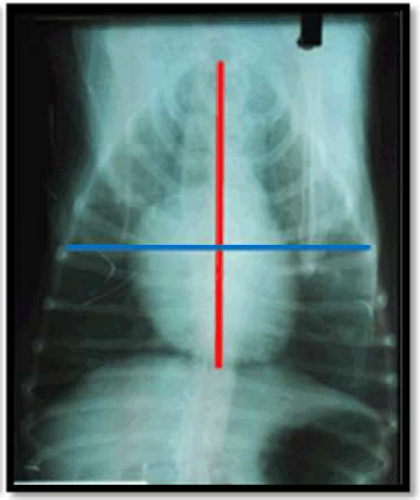

C

Figura 3. Toma de medidas de ancho (línea azul) y largo (línea roja) del tórax para la clasificación en (A) estrecho (relación largo: ancho de 3:2); (B) normal (relación largo: ancho de 3:2.5); (C) en barril (relación largo: ancho de 3:3), según la vista dorsoventral alineada (Control)

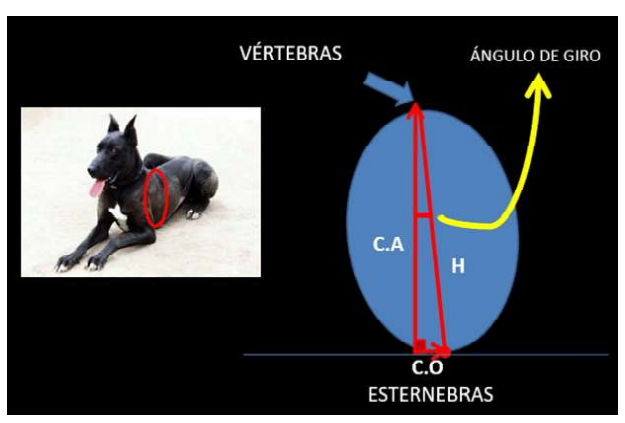

Figura 4. Metodología para hallar el ángulo de giro en la vista radiográfica dorsoventral girada, mediante el uso de la muestra control (dorsoventral alineada) y vista latero lateral derecha, donde: $\mathrm{CO}$, distancia entre vértebra y esternebra, según vista DV girada; $H$, distancia entre esternebras y vértebras según vista LLD

que dará el valor del ángulo en radianes y luego el valor es convertido en grados mediante la función grados de Excel (GRADOS). Los ángulos obtenidos se distribuyeron en un grupo con $0.1^{\circ}$ a $6^{\circ}$ de giro y otro con $6^{\circ}$ a $12^{\circ}$ grados de giro; ambos con sus respectivas muestras control, como se muestra en la Figura 4. La determinación de los grupos se realizó en base a la cantidad de muestras obtenidas con diferentes ángulos obtenidos al azar con agrupación equitativa.

\section{Ubicación del desplazamiento}

Se evaluó la zona del desplazamiento, ya sea en la porción craneal o caudal del tórax del paciente y la dirección (hacia el lado izquierdo o lado derecho), tomando como referencia la columna vertebral del paciente, puesto que dichas variantes pueden afectar a la región cardiaca donde se presenta el aumento, en comparación al aumento presentado en la placa control. La comparación del aumento entre las placas giradas y los controles se realiza para cada cámara cardiaca por separado.

Como se puede observar en las Figura 5 , se obtuvieron como mínimo tres vistas por sujeto muestreado donde se tiene la vista LLD, la vista DV alineada y la vista DV no alineada, la cual puede presentar el giro, tanto en la parte craneal del sujeto como en la parte caudal o en ambas partes. 


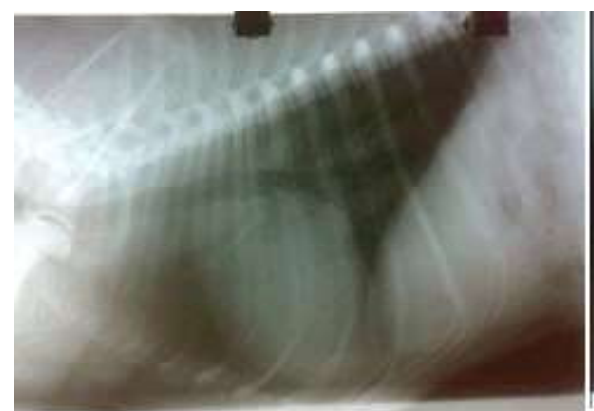

(A)

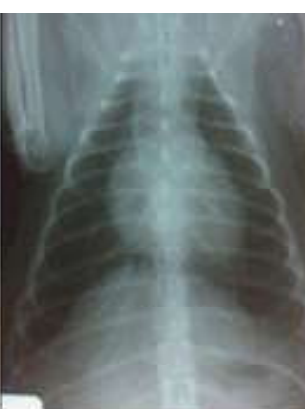

(B)

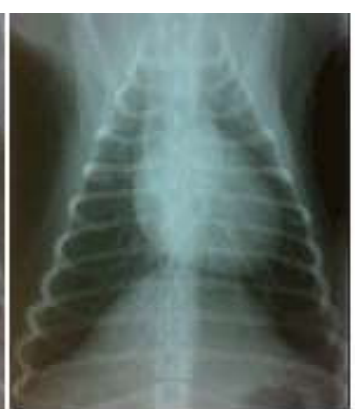

(C)

Figura 5. Paciente canino, Chihuahua, macho, 5 años (Clínica de Animales Menores, FMVUNMSM). (A) Vista radiográfica latero lateral derecha. (B) Vista dorsoventral alineada. (C) Vista dorsoventral no alineada que pertenece a grupo muestral con giro de $6^{\circ}$ a $12^{\circ}\left(6.37^{\circ}\right.$ de giro). Nótese el giro hacia el lado derecho en la porción caudal del cuerpo del paciente

\section{Terminología del desplazamiento}

Debido a que la comparación de los parámetros cardiacos se realizó para cada cámara cardiaca y que estos dependían de la ubicación del desplazamiento, se hizo la clasificación con fines prácticos de la siguiente manera: $\mathrm{D} / \mathrm{AR}$ representa el giro hacia el lado derecho en la porción craneal del tórax; $\mathrm{D} /$ $\mathrm{AB}$, hacia el lado derecho en la porción caudal del tórax; I/AR, giro hacia el lado izquierdo en la porción craneal del tórax; $\mathrm{I} / \mathrm{AB}$, giro hacia el lado izquierdo en la porción caudal del tórax; $\mathrm{D} / \mathrm{AR}-\mathrm{AB}$, giro hacia el lado derecho, tanto en la porción craneal como en la porción caudal del tórax; I/AR-AB, giro hacia el lado izquierdo, tanto en la porción craneal como en la porción caudal del tórax.

\section{Evaluación Estadística}

Los datos obtenidos en cada grupo muestral fueron analizados de acuerdo a cada cámara cardiaca por separado utilizando el programa Stata mediante la prueba de McNemar.

\section{Resultados}

De las 53 muestras, 23 se obtuvieron con rango de giro entre $0.1^{\circ}$ a $6^{\circ}$ y 30 con rangos entre $6^{\circ}$ a $12^{\circ}$ de giro. Los resultados del análisis estadístico indican que existe una probabilidad de Chi cuadrado para la aurícula derecha de 0.1025 y de 0.6547 para la aurícula izquierda, mientras que para el ventrículo derecho fue de 0.1573 y de 0.3173 para el ventrículo izquierdo, entre ambos métodos diagnósticos $(\mathrm{p}>0.05)$. Esto indica una asociación entre las placas DV con grado de giro de hasta $6^{\circ}$ y las placas DV alineadas para todas las cámaras cardiacas, indicando que pueden ser usadas para diagnóstico cardiológico, como se puede observar en la Figura 6.

En el grupo de $6^{\circ}$ a $12^{\circ}$ grados de giro se obtuvo una probabilidad de 0.0196 al evaluar la aurícula derecha y de 0.0578 para la aurícula izquierda, en tanto que para el ventrículo derecho fue de 0.0114 y de 0.0126 para el ventrículo izquierdo $(\mathrm{p}<0.05)$, indicando que las placas con grados de giro mayor a $6^{\circ}$ 

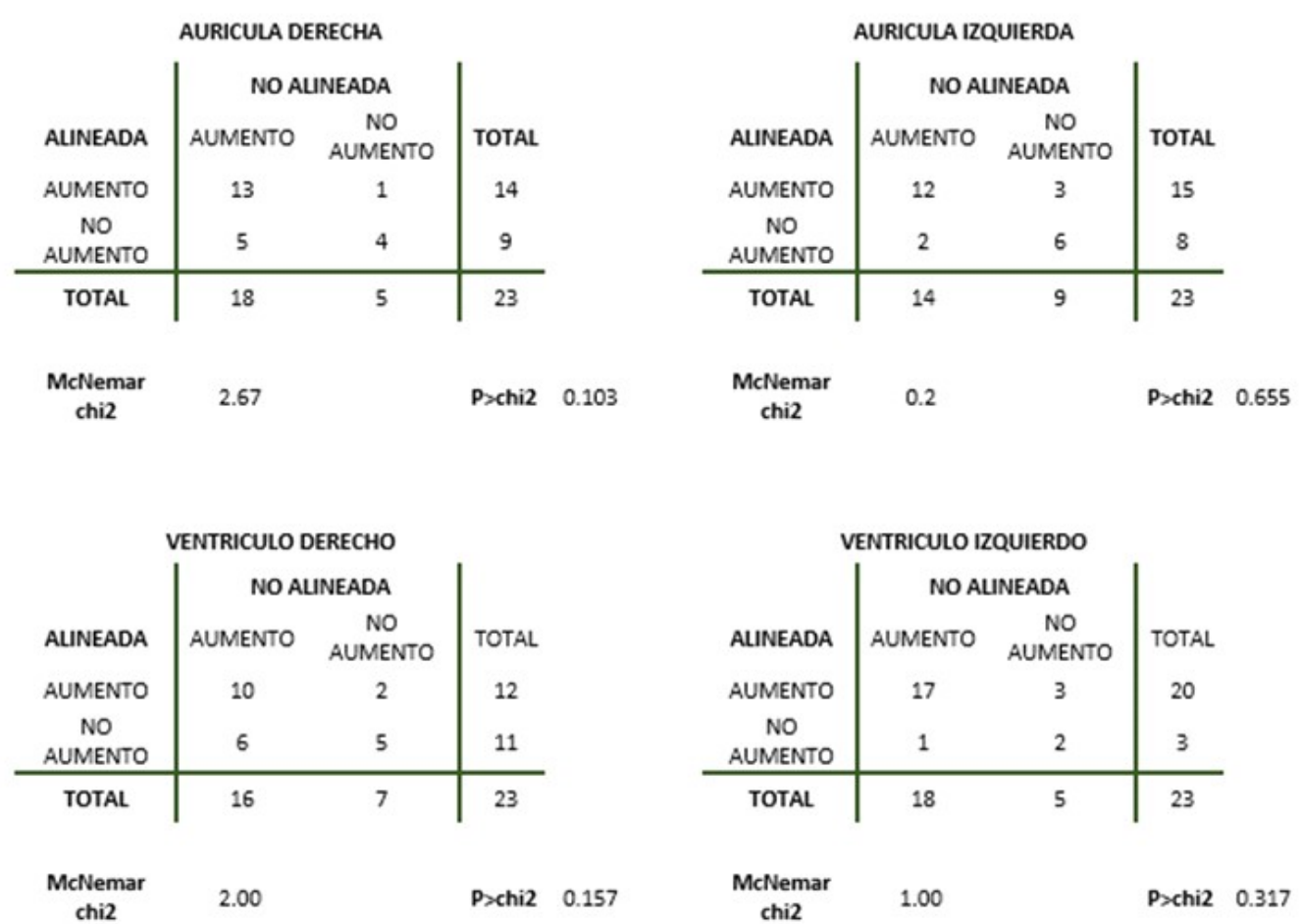

Figura 6. Resultados obtenidos mediante el uso de la prueba estadística McNemar para cada cámara cardiaca en el grupo de placas no alineadas dorsoventrales con $0.1^{\circ} \mathrm{a} 6^{\circ}$ de giro

no pueden ser usadas para diagnóstico cardiológico, como se puede observar en la Figura 7.

Al analizar la dirección de giro y la coincidencia de resultados con el grupo control se obtuvo un mayor porcentaje de coincidencia en cuanto a diagnóstico para el grupo de muestras que giraron hacia el lado derecho y en la porción craneal del tórax $(\mathrm{D} / \mathrm{AR}) \mathrm{de}$ $75 \%$ y en el grupo de muestras que giraron hacia el lado izquierdo y en la porción caudal del tórax (I/AB) de $75 \%$ en el grupo con giro de $0.1^{\circ}$ a $6^{\circ}$. Mientras que en el grupo con giro entre $6^{\circ}$ a $12^{\circ}$, el porcentaje de coincidencia de diagnóstico para giro hacia la derecha y craneal (D/AR) fue de $100 \%$ y para el giro hacia la izquierda y caudal (I/AB) fue de $78.3 \%$, como se observa en el Cuadro 1 .

La conformación torácica se clasificó con base a la proyección DV para determinar si el diagnóstico de la proyección girada se ve influenciado por el tipo de tórax. Se encontró que la coincidencia de diagnóstico entre las vistas control y las vistas DV giradas o grupos con grado de giro de $0.1^{\circ}$ a $6^{\circ} \mathrm{y}$ de $6^{\circ}$ a $12^{\circ}$ fue siempre menor en aquellos pacientes con tórax en barril (Figura 8); además, la coincidencia disminuye conforme el grado de giro aumenta (en las hembras del grupo con grado de giro de $6^{\circ}$ a $12^{\circ}$ llega a ser nula la coincidencia).

\section{Discusión}

Cada cámara o parte del corazón fue evaluada por separado, debido a que existía la posibilidad de que la radiografía control no presentara aumento de una de las partes del corazón y en la radiografía DV girada se presentase aumento de dicha parte, de manera que al ser evaluadas mediante la prueba estadística de McNemar cada parte del cora- 

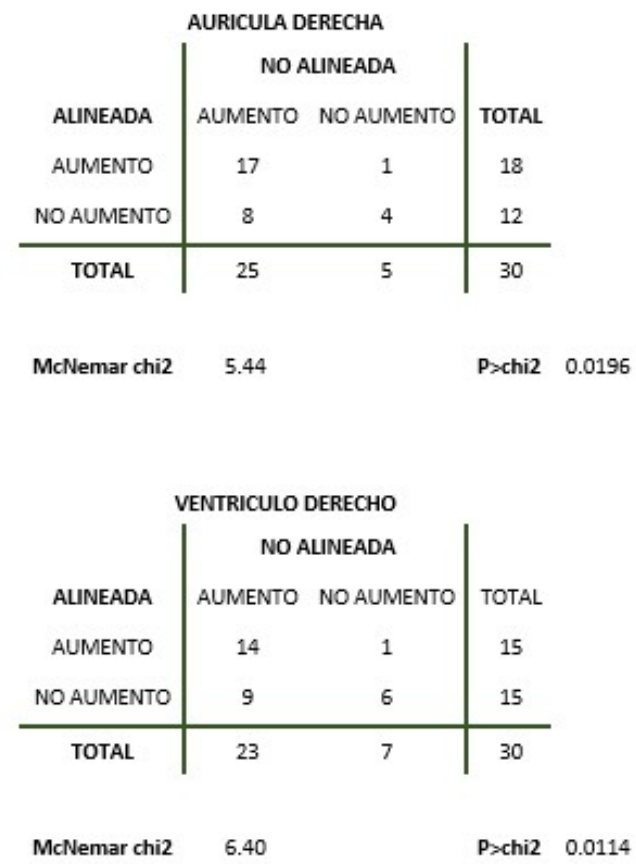
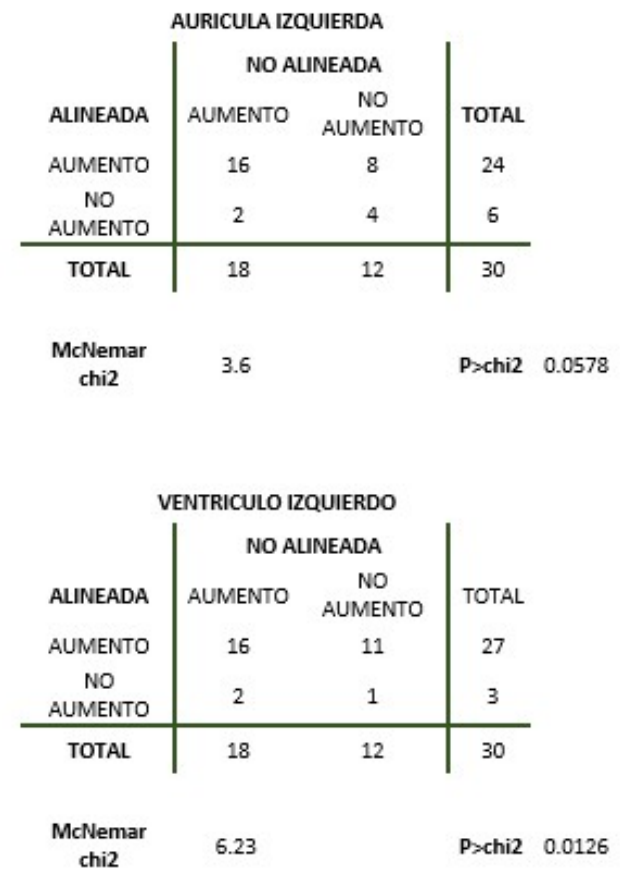

Figura 7. Resultados obtenidos mediante el uso de la prueba estadística McNemar para cada cámara cardiaca en el grupo de placas no alineadas dorsoventrales con $6^{\circ}$ a $12^{\circ}$ de giro.
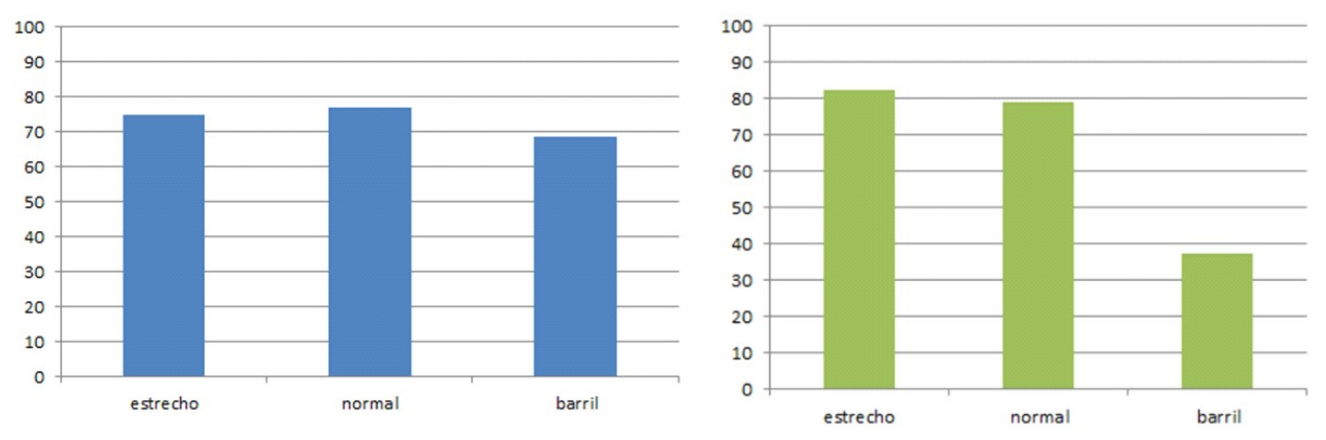

Figura 8. Porcentaje de coincidencia de diagnóstico del grupo muestral con el grupo control teniendo en cuenta el tipo de tórax según vista dorsoventral. Izquierda: Con grado de giro de $0.1^{\circ}$ a $6^{\circ}$; Derecha: Con grado de giro de $6^{\circ}$ a $12^{\circ}$.

zón debía superar el valor de probabilidad de 0.05 para indicar que sigue una distribución normal y se corrobore la hipótesis nula. Así, los resultados demuestran una probabilidad mayor a 0.05 a nivel de todas las cámaras cardiacas para el grupo de radiografías DV con grado de giro entre $0.1^{\circ}$ a $6^{\circ}$ y no para el grupo de placas DV con rango de giro entre $6^{\circ}$ a $12^{\circ}$. Por lo que se puede decir que al no haber significancia estadística entre el grupo control y el grupo con $0.1^{\circ}$ a $6^{\circ}$ de giro, los diagnósticos de radiografías DV con grado de giro menor a $6^{\circ}$ son aceptables. 
Cuadro 1. Porcentaje de coincidencia de diagnóstico entre las muestras control y muestras problema de acuerdo con la dirección del giro ${ }^{1}$

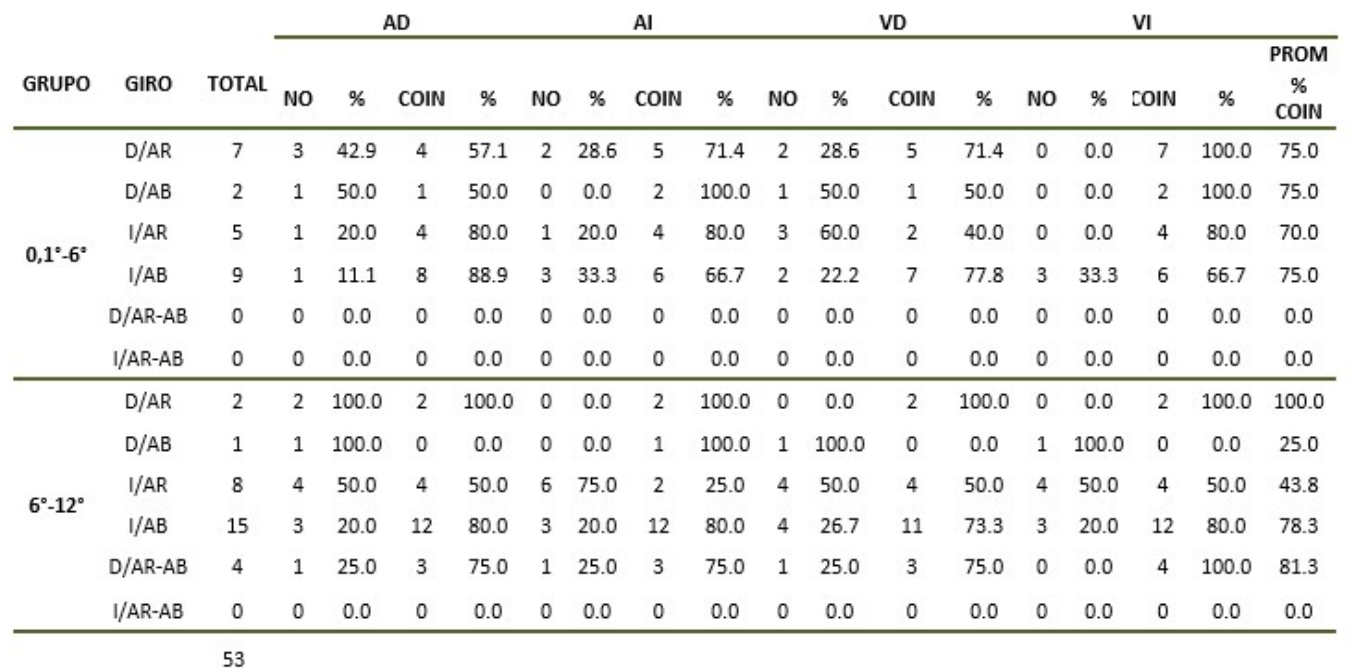

${ }^{1} D / A R$ representa el giro hacia el lado derecho en la porción craneal del tórax; $D / A B$, hacia el lado derecho en la porción caudal del tórax; I/AR, giro hacia el lado izquierdo en la porción craneal del tórax; I/AB, giro hacia el lado izquierdo en la porción caudal del tórax; $D / A R-A B$, giro hacia el lado derecho tanto en la porción craneal como en la porción caudal del tórax; I/AR-AB, giro hacia el lado izquierdo tanto en la porción craneal como en la porción caudal del tórax. NO, no coincidencia en diagnóstico y COIN, coincidencia en el diagnóstico

Holmes et al. (1985) realizaron un estudio comparativo de la silueta cardiaca de placas no alineadas ventrodorsales con la de placas alineadas ventrodorsales en 34 perros Beagle, encontrando que la amplitud del lado derecho del corazón era mayor o aumentaba y el lado izquierdo disminuía en los pacientes peor posicionados. Además, encontraron que no había diferencia significativa entre grupos al comparar la proporción cardiotorácica y el lado derecho del corazón y, por lo tanto, determinaron que no debían descartarse las placas ventrodorsales giradas. Sin embargo, hay que recalcar que dicho estudio usó como referencia placas ventrodorsales, que como ya se mencionó previamente, no son del todo confiables al momento de realizar un estudio cardiológico, debido a que el corazón al no encontrarse suspendido sobre su ápice se recuesta dando un aspecto más alargado y puede enmascarar el aumento cardiaco de alguno de sus lados (BSAVA, 2000; Thrall, 2012).
Por otro lado, el presente estudio, en comparación con el trabajo de Holmes et al. (1985), cuenta con la placa alineada de cada muestra DV girada, de allí que se puede realizar un análisis comparativo más preciso de los diagnósticos y parámetros obtenidos. Así mismo, dicho estudio realizó la medición de rotación en base al desplazamiento de las apófisis espinosas y si sobresalían de los límites de los cuerpos vertebrales, sin especificar el número de apófisis espinosas que debían ser evaluadas.

El método usado para calcular el ángulo de giro en el presente estudio obtuvo resultados similares a los resultados obtenidos por Hamlin (1968) quién tomó como referencia la profundidad del tórax, así como la distancia entre las vértebras y esternebras de la proyección DV no alineada, haciendo uso de un nomógrafo para calcular el ángulo de giro. La variante utilizada en el presente estudio 
permite el cálculo del ángulo de giro de forma simplificada obteniendo los mismos resultados que en la metodología realizada por dicho autor, lo que lo hace un método confiable y rápido para el cálculo del ángulo de giro en proyecciones DV de tórax no alineadas. Se puede reducir el estrés al paciente cardiópata y la dosis de radiación recibida por el tecnólogo o radiólogo al reducir el número de repeticiones para la obtención de una placa DV alineada, al aceptar o permitir hasta $6^{\circ}$ de giro en dicha vista radiográfica.

De las 23 muestras pertenecientes al grupo con rango de giro de $0.1^{\circ}$ a $6^{\circ}, 14$ fueron de pacientes asintomáticos, cuatro presentaron problemas o signos respiratorios (tos, disnea, agitación o cianosis), siete presentaron soplo y/o arritmia, dos presentaron inapetencia, uno presentó tremores y uno presentaba convulsiones. Esto indica que en numerosas ocasiones, el propietario no percibe sintomatología alguna en el paciente, siendo otra la causa del motivo de la consulta, donde el problema cardiaco es un hallazgo accidental por parte del Médico Veterinario (Kittleson y Kienle, 2000).

Se tuvieron 260 casos de perros diagnosticados con alguna cardiopatía en la UNMSM durante el periodo 2007-2009 (Calderón et al., 2014), donde en muchos casos se complementó el estudio radiológico con la ecocardiografía a fin de eliminar parte de la subjetividad del examen radiológico (Agut, 2014). Es por eso que muchos radiólogos veterinarios se reservaban la emisión del diagnóstico radiológico hasta tener el diagnóstico final de la ecocardiografía, a excepción del aumento de aurícula izquierda, donde la radiografía es igual de exacta que la ecocardiografía (Kittleson y Kienle, 2000).

\section{Conclusiones}

- El método usado para medir el ángulo de giro en el presente estudio es aceptable, por lo que podría ser usado en estudios posteriores, siendo sencillo y rápido de realizar en comparación con otros métodos.

- Placas dorsoventrales con grado de giro de $0.1^{\circ}$ a $6^{\circ}$ son aceptables para diagnóstico.

\section{Literatura Citada}

1. Aguilar S. 2005. Fórmulas para el cálculo de la muestra en investigaciones de salud. Salud en Tabasco 11:333-338.

2. Agut A. 2014. Diagnóstico por imagen en pequeños animales. Barcelona, España: Ed Multimédica. $584 \mathrm{p}$.

3. Álvarez ET, Peralta DL, García M, Cavagión L, Larrieu E, Ferrán A. 2007. Enfermedades y lesiones laborales en médicos veterinarios de Argentina. Ciencia Veterinaria 9: 49-67.

4. Atkins C, Bonagura J, Ettinger S, Fox P, Gordon S, Haggstrom J, Hamlin $\boldsymbol{R}$, et al. 2009. Guidelines for the diagnosis and treatment of canine chronic valvular heart disease. J Vet Intern Med 23: 1142-1150. doi: 10.1111/j.19391676.2009.0392.x

5. [BSAVA] British Small Animal Veterinary Association. 2000. Manual de medicina y cirugía cardiorrespiratoria en pequeños animales. España: Harcourt Brace. 502 p.

6. Buchanan JW. 2000. Vertebral scale system to measure heart size in radiographs. Vet Clin N Am-Small 30: 379-393. doi: 10.1016/S0195-5616(00)50027-8

7. Bushong SC. 2013. Manual de radiología para técnicos: física, biología y protección radiológica. $10^{\circ}$ ed. Texas, EEUU: Elsevier. 664 p.

8. Calderón K, Dávila R, Gavidia C. 2014. Casuística de enfermedades cardiacas en caninos de la clínica de animales menores de la Facultad de Medicina Veterinaria, Universidad Nacional Mayor de San Marcos, durante el periodo 2007-2009. Rev Inv Vet Perú 25: 399-405. doi: 10.15381/rivep.v25i3.10118 
9. Hamlin RL. 1968. Analysis of the cardiac silhouette in dorsoventral radiographs from dogs with heart disease. J Am Vet Med Assoc 153: 1446-1460.

10. Hartung K. 1992. Radiation exposure of the hands and feet during $\mathrm{X}$-ray studies in small animals. Tierarztl Prax 20: 187-193.

11. Holmes R, Smith F, Lewis R, Kern D. 1985. The effects of rotation on the radiographic appearance of the canine cardiac silhouette in dorsal recumbency. Vet Radiol Ultrasoun 26: 98-101. doi: 10.1111/j.1740-8261.1985.tb01390.x

12. [IPEN] Instituto Peruano de Energía Nuclear. 2013. Resolución Presidencial sobre Requisitos de Protección Radioló- gica en Diagnóstico Médico con Rayos X. IR.003.2013. 19 p.

13. Kittleson M, Kienle R. 2000. Medicina cardiovascular de pequeños animales. $2^{\circ} \mathrm{ed}$. Barcelona, España: Ed Multimédica. 675 p.

14. Massey JB. 1971. Manual de dosimetría en radioterapia. Guía práctica para la revisión y calibración del equipo utilizado en radioterapia externa. Viena, Austria: OIEA. $158 \mathrm{p}$.

15. Tilley L, Smith F, Oyama M, Sleeper M. 2008. Manual of canine and feline cardiology. $4^{\circ}$ ed. Missouri, EEUU: Saunders. $443 \mathrm{p}$.

16. Thrall D. 2012. Textbook of veterinary diagnostic radiology. $6^{\circ}$ ed. EEUU: Universidad de Carolina del Norte. 864 p. 